tions. As human numbers rise ever closer to the point of being unsustainable, it is not surprising that this valuable resource is coming under closer scrutiny.

Malcolm Coe is at 39 Sandfield Road, Headington, Oxford OX3 7RN, UK.

\section{Dip into the century's technological past}

\section{Visions of Technology}

by Richard Rhodes

Simon \& Schuster: 1999. 400 pp. \$30, £20

\section{Henry Petroski}

This anthology opens abruptly, but appropriately, with a view from the late 1920s reminding us of words that were not part of the American vocabulary at the turn of the century - radio, movie, tractor, aviator, income tax, insulin, relativity, quantum theory, behaviourism. The list goes on, and we are engaged immediately by the realization that, throughout the century, most of us have been as close as a generation or two, and at most a few generations away from a very different world of things and ideas.

This collection of excerpts from a broad range of thinking and opinion about technology throughout the twentieth century makes for fast, enjoyable and sometimes fascinating reading. There are first-hand reports of their technologies from the likes of Guglielmo Marconi, Wilbur Wright, Henry Ford and George Eastman; opinions and observations from theorizers and visionaries such as Henry Adams, Thorstein Veblen, H. G. Wells and Robert Frost. The authority and stature of these representatives of the earliest years of the century give a fair idea of the quality and breadth of the collection, whose second half, not surprisingly, is filled with discussions of nuclear weapons, the two-cultures debate, the environment and computers. The selections are always apposite, seldom too long, and almost never uninteresting. They average fewer than two pages in length, with manyless than half a page.

In addition to textual excerpts, brief quotations, photos, diagrams and graphs are scattered throughout the book, but in general these are not as informative or satisfying as the text. In fact, many of the graphs seem arbitrarily cut off well before the end of the century, frustrating to the reader. One graph, appearing with a 1927 piece by Aldous Huxley on comfort, compares numbers of telephone calls made and pieces of mail sent per capita per year, but is inexplicably curtailed in the mid-1970s. Another graph showing the growth in numbers of personal computers in use from 2 million in 1981 to 38 million in 1987 makes the reader wonder how many are in use today.
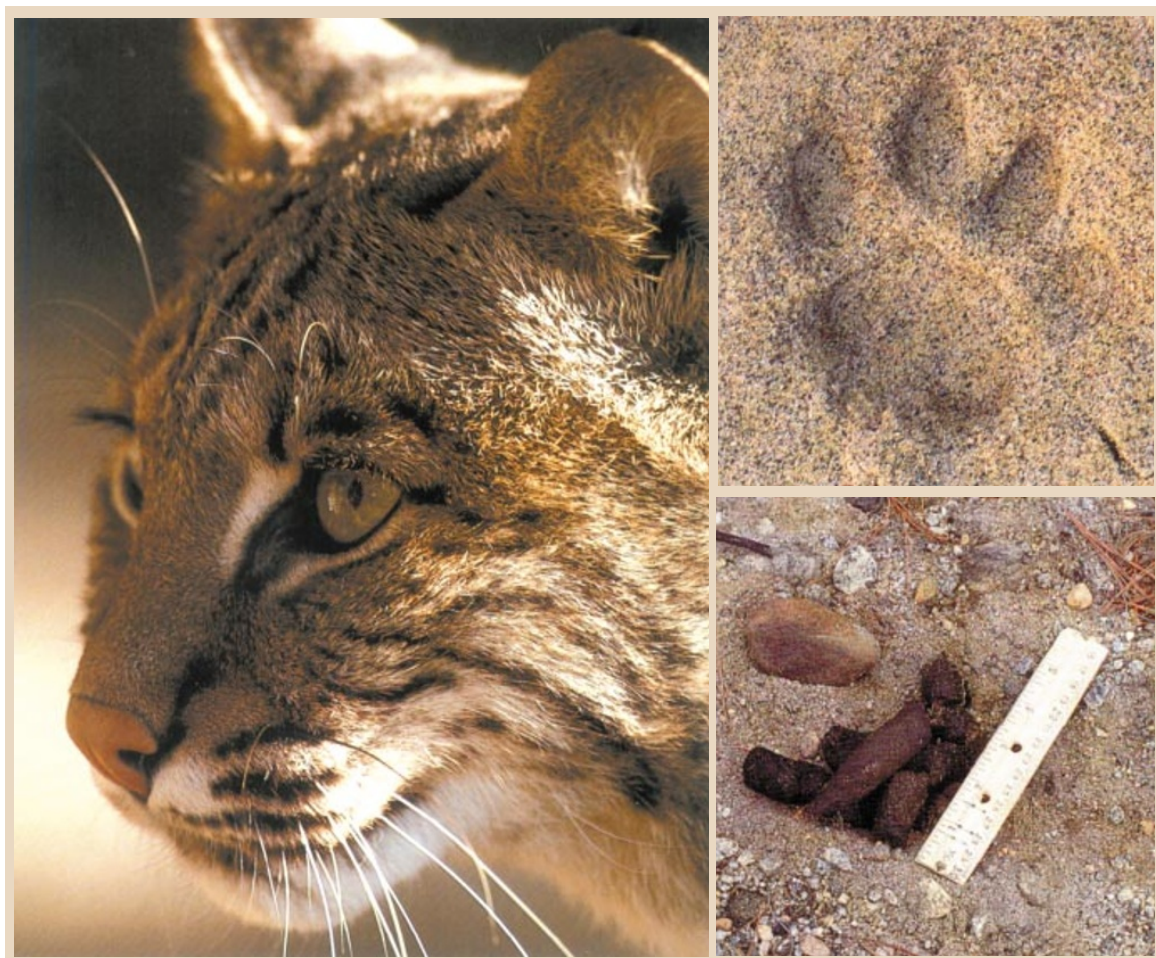

\title{
A real whodunnit
}

How many of us, from looking at the two items on the right - a paw track and a snatch of faecal scat - would recognize them as the signature of the bobcat on the left? Paul Rezendes, nature photographer, tracking expert and author of Tracking and the Art of Seeing: How to Read Animal Tracks and Sign (HarperCollins, \$24),

As with all anthologies, especially wideranging ones, any reader will bring his or her own expectations to the book and finish it with a list of addenda and concerns. Engineering as a profession is treated explicitly in fewer than six selections, including one from Herbert Hoover's mid-century memoirs. The editor's introduction to the piece is a bit misleading, however, in stating that "engineering was just becoming a profession at the turn of the century". In spite of the anecdotes of Hoover and others to the contrary, engineering as a profession was, in fact, established well before the beginning of the twentieth century: the American Society of Civil Engineers was founded in 1852 and the British Institution of Civil Engineers some decades before that.

In fact, it is the seemingly ambivalent role of Richard Rhodes as editor of this collection that may be its most distracting and frustrating quality, although the perception may be a result of the book's design. Throughout, the left-hand running head, set in the centre of the left margin and reading vertically over horizontal page numbers, places the name of RICHARD RHODES beside the continuations of quotations from the likes of Edwin Land, Elting Morison, B. F. Skinner and John von Neumann, whose names are not carried would have little difficulty. Bobcats aside, Rezendes' book describes the behaviour and habitats of more than $\mathbf{5 0}$ animal species, and how they can be identified, not only from their tracks and droppings, but from trail patterns, scratches and clues that most of us would blunder blithely past.

over as running heads.

Some of Rhodes's brief introductions to the selections are helpful, as when he tells us how Gordon E. Moore, one of the founders of the Intel Corporation, came to write the excerpted article that gave us the law relating to the growth rate of integrated circuits. Other selections, however, have such brief introductions that they are almost useless. Thus, in one note to a selection from 1971, Daniel C. Drucker is identified simply as "a dean of engineering". The reader unfamiliar with Drucker's career will not know that he was dean at one of the nation's premier engineering schools, the University of Illinois at Urbana-Champaign. Other authors, generally less well known than Drucker, are left completely unidentified. Perhaps even more disappointing is the fact that the reader is not told the source, occasion or context of the vast majority of the selections. This is an anthology that excels in its selections but that is wanting in its editorial and visual design.

Henry Petroski is in the Department of Civil and Environmental Engineering, Duke University, Durham, North Carolina 27708-0287, USA. His book on the history of the book shelf, The Book on the Bookshelf, will be published by Knopf in September. 